\title{
Compressive Detection Using Sub-Nyquist Radars for Sparse Signals
}

\author{
Ying Sun, ${ }^{1}$ Jianjun Huang, ${ }^{1}$ Jingxiong Huang, ${ }^{1,2}$ Li Kang, ${ }^{1}$ Li Lei, ${ }^{1}$ and Yi Tang ${ }^{1}$ \\ ${ }^{1}$ ATR Key Laboratory, Shenzhen University, Shenzhen, Guangdong 518060, China \\ ${ }^{2}$ Air Defense Forces Academy, Zhengzhou 450052, China \\ Correspondence should be addressed to Ying Sun; baobaoshu126@126.com
}

Received 30 May 2016; Revised 15 August 2016; Accepted 6 September 2016

Academic Editor: Lorenzo Crocco

Copyright (c) 2016 Ying Sun et al. This is an open access article distributed under the Creative Commons Attribution License, which permits unrestricted use, distribution, and reproduction in any medium, provided the original work is properly cited.

\begin{abstract}
This paper investigates the compression detection problem using sub-Nyquist radars, which is well suited to the scenario of high bandwidths in real-time processing because it would significantly reduce the computational burden and save power consumption and computation time. A compressive generalized likelihood ratio test (GLRT) detector for sparse signals is proposed for subNyquist radars without ever reconstructing the signal involved. The performance of the compressive GLRT detector is analyzed and the theoretical bounds are presented. The compressive GLRT detection performance of sub-Nyquist radars is also compared to the traditional GLRT detection performance of conventional radars, which employ traditional analog-to-digital conversion (ADC) at Nyquist sampling rates. Simulation results demonstrate that the former can perform almost as well as the latter with a very small fraction of the number of measurements required by traditional detection in relatively high signal-to-noise ratio (SNR) cases.
\end{abstract}

\section{Introduction}

Application of compressed sensing/sampling (CS) [1-4] in radar signal processing has recently caught the attention of many researchers. Modern radar systems often operate with high bandwidths. According to the bandpass sampling theorem [5], it is necessary to sample a signal at the Nyquist rate in order to avoid aliasing. Therefore, it would lead to huge amounts of data, which require very big storage capacity, cause high power consumption, and make the subsequent real-time digital signal processing (DSP) difficult. Due to the sparsity of radar target signal, CS techniques can be applied to radar systems to alleviate the pressure of signal acquisition. Consequently, many works have been carried out for radar based on CS [6-13]. The paper [6] investigates an $\mathrm{A} / \mathrm{D}$ converter that operates at a low sampling rate rather than at its high Nyquist rate and presents a rather preliminary approach to CS synthetic aperture radar (SAR) imaging. In [7], a stylized CS radar is proposed exploiting the sparsity of the radar signal in the time-frequency plane by use of suitable waveforms (called the Alltop sequence). References $[14,15]$ extend further CS into multiple input multiple output
(MIMO) radar to attain more efficient processing for target detection and parameters estimation. Paper [14] sketches the CS application for distributed MIMO radar and investigates waveforms optimized problem in this environment. The work in [15] proposes a simulated annealing (SA) algorithm based on waveform design method which can produce the waveform with smaller target response correlation contrasted to the Alltop sequence. Recovery methods are indispensable in these works. Current reconstruction methods include using greedy algorithms such as orthogonal matching pursuit (OMP) [16] and solving the convex problem such as the basis pursuit (BP) $[1,2]$. However, these algorithms involve iterative optimization procedures and are thus computationally expensive for long signals. For this reason, the recent research report [17] studied a novel signal processing approach directly based on the compressive measurements. Specifically, the detection problem based on compressive measurements has been also discussed in [18-20]. Paper [18] proposed jointly compressive signal target detection and parameters estimator in radar without signal reconstruction. A subspace compressive GLRT (SSC-GLRT) detector is proposed in [19]. The work in [20] is an extension of the former, which applies 
the SSC-GLRT detector to MIMO airborne radar systems. However, reconstruction algorithms are still required in the SSC-GLRT detector to approximately estimate the signal sparsity model [19], which eventually will bring about huge computational burden.

Distinct from the generalized CS radar in [21, 22], this paper focuses on the compressive detection problem using sub-Nyquist radars [23-25]. Sub-Nyquist radars allow sampling of radar signals at rates much lower than Nyquist, which has been demonstrated by real-time analog experiments in hardware [23]. Sub-Nyquist techniques allow significant reduction in sampling rate, implying potential power saving, and hence gaining substantial storage capacity reduction for subsequent processing. More precisely, our goal is tantamount to determining whether any target exists from compressive measurements obtained from subNyquist radars without reconstructing the signal involved. Hence, a compressive GLRT detector using sub-Nyquist radars for sparse signal is proposed, which employs the direct multichannel sampling technique [24] and does not adopt any reconstruction algorithms so that it can reduce the computational burden and save power consumption and computation time. The performance of the compressive GLRT detector is analyzed and the theoretical bounds are provided. The compressive GLRT detection performance of sub-Nyquist radars is also compared to the traditional GLRT detection performance of conventional radars, which employs traditional analog-to-digital conversion (ADC) at Nyquist sampling rates. Simulation results demonstrate that the former can perform almost as well as the latter with a very small fraction of the number of measurements required by traditional detection in relatively high signal-to-noise ratio (SNR) cases. That is to say, it is possible to achieve comparable performance with less computational load and less computation time for sub-Nyquist radar in some certain cases.

The rest of the paper is organized as follows. Section 2 builds sub-Nyquist radar signal model. Section 3 presents the compressive GLRT detector for sub-Nyquist. In Section 4, we analyze the compressive GLRT detection performance and provide its theoretical bounds. Several numerical examples are presented in Section 5 to verify the correctness of the theoretical deviations. We conclude in Section 6.

\section{Signal Model of Sub-Nyquist Radars}

Consider a sub-Nyquist radar, in which a signal pulse is transmitted. The round trip delay $\tau$ from the transmitter to the target and back is related to the range $R$ as $\tau=2 R / c$, where $c$ is the speed of propagation. If $s(t)$ is the transmitted signal, a simple model for the received continuous waveform can be written as

$$
r(t)=\alpha s(t-\tau)+w(t), \quad 0 \leq t \leq T .
$$

The scalar $\alpha$ represents the unknown complex target reflectivity. $w(t)$ is a zero mean wide-sense stationary complex Gaussian white noise with autocorrelation $r_{w}(s)=\sigma^{2} \delta(s)$. The transmitted signal pulse is assumed to be nonzero over the interval $\left[0, t_{p}\right]$ with continuous-time Fourier transform

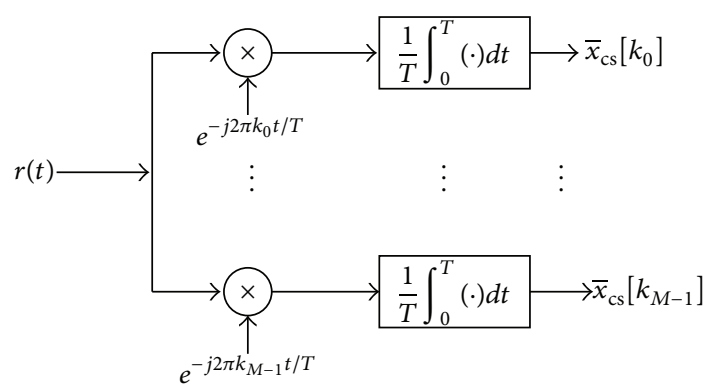

FIGURE 1: Multichannel direct sampling of the Fourier series coefficients, from [24].

(CTFT) $\bar{s}(f)=\int_{-\infty}^{\infty} s(t) e^{-j 2 \pi f t} d t$. Additionally, we assume that $\bar{s}(f)$ has negligible energy at frequencies beyond $B \mathrm{~Hz}$. If the maximum time delay is $\tau_{\text {max }}$, then the observation interval is chosen to include the entire signal by letting $T=t_{p}+\tau_{\max }$. The continuous received waveform can be sampled at the sub-Nyquist rate using the direct multichannel sampling scheme described in [24], as illustrated in Figure 1.

The analog input $r(t)$ is split into $M$ channels, where in each channel $k_{m}$ it is mixed with the harmonic signal $e^{-j 2 \pi k_{m} t / T}$ integrated over $T$ and then sampled. Consequently, we obtain the corresponding vector of Fourier coefficients

$$
\overline{\mathbf{x}}_{c s}=\left[\bar{x}_{c s}\left[k_{0}\right], \bar{x}_{c s}\left[k_{1}\right], \ldots, \bar{x}_{c s}\left[k_{M-1}\right]\right]^{T},
$$

where

$$
\bar{x}_{c s}\left[k_{m}\right]=\frac{1}{T} \int_{0}^{T} r(t) e^{-j 2 \pi k_{m} t / T} d t
$$

where $m=0,1, \ldots, M-1$. Taking (1) into (3) yields to

$$
\bar{x}_{c s}\left[k_{m}\right]=\frac{\alpha}{T} \bar{s}_{c s}\left[k_{m}\right] e^{-j 2 \pi k_{m} \tau / T}+\bar{w}_{c s}[m],
$$

where

$$
\begin{aligned}
& \bar{s}_{c s}\left[k_{m}\right]=\int_{0}^{T} s(t) e^{-j 2 \pi k_{m} t / T} d t, \\
& \bar{w}_{c s}[m]=\frac{1}{T} \int_{0}^{T} w(t) e^{-j 2 \pi k_{m} t / T} d t
\end{aligned}
$$

is a zero mean complex random variable with variance

$$
\begin{aligned}
E & {\left[\left|\bar{w}_{c s}[m]\right|^{2}\right] } \\
& =\frac{1}{T^{2}} \int_{0}^{T} d t \int_{0}^{T} E\left[w(t) w^{*}\left(t^{\prime}\right)\right] e^{-j 2 \pi k_{m}\left(t-t^{\prime}\right) / T} d t^{\prime} \\
& =\frac{1}{T^{2}} \int_{0}^{T} d t \int_{0}^{T} \sigma^{2} \delta\left(t-t^{\prime}\right) e^{-j 2 \pi k_{m}\left(t-t^{\prime}\right) / T} d t^{\prime} \\
& =\frac{1}{T^{2}} \sigma^{2} \int_{0}^{T} d t=\frac{\sigma^{2}}{T} .
\end{aligned}
$$


We can then write (4) in vector form as

$$
\overline{\mathbf{x}}_{c s}=\frac{\alpha}{T} \Lambda \mathbf{d}(\tau)+\overline{\mathbf{w}}_{c s},
$$

where

$$
\begin{aligned}
\mathbf{d}(\tau) & =\left[e^{-j 2 \pi k_{0} \tau / T}, e^{-j 2 \pi k_{1} \tau / T}, \ldots, e^{-j 2 \pi k_{M-1} \tau / T}\right]^{T} \\
\overline{\mathbf{w}}_{c s} & =\left[\bar{w}_{c s}\left[k_{0}\right], \bar{w}_{c s}\left[k_{1}\right], \ldots, \bar{w}_{c s}\left[k_{M-1}\right]\right]^{T},
\end{aligned}
$$

and $\boldsymbol{\Lambda}=\operatorname{diag}\left\{\overline{\mathbf{s}}_{c s}\right\}$, where

$$
\overline{\mathbf{s}}_{c s}=\left[\bar{s}_{c s}\left[k_{0}\right], \bar{s}_{c s}\left[k_{1}\right], \ldots, \bar{s}_{c s}\left[k_{M-1}\right]\right]^{T} .
$$

Under the $H_{1}$ hypothesis (target present) and $H_{0}$ hypothesis (target absent), respectively, the detection problem can be represented as

$$
\begin{aligned}
& H_{0}: \overline{\mathbf{x}}_{c s}=\overline{\mathbf{w}}_{c s} \\
& H_{1}: \overline{\mathbf{x}}_{c s}=\frac{\alpha}{T} \Lambda \mathbf{d}(\tau)+\overline{\mathbf{w}}_{c s},
\end{aligned}
$$

where $\overline{\mathbf{w}}_{c s}$ is zero mean complex Gaussian white noise with the correlation matrix $\left(\sigma^{2} / T\right) \mathbf{I}_{M \times M}$.

\section{Compressive GLRT Detector for Sub-Nyquist Radars}

Using the signal models defined in (10), we derive the detector for the sub-Nyquist sampling scheme based upon the generalized likelihood ratio test (GLRT). We assume that, in the cell-under-test, there is either a single target with a delay $\tau$ or no target at all.

For sub-Nyquist radar, the probability density function (PDF) of the compressive measurements vector conditioned on the hypotheses and parameters can be written [26] as

$$
\begin{aligned}
& f\left(\overline{\mathbf{x}}_{c s} \mid \alpha, \tau, H_{1}\right)=\frac{1}{\pi^{M}\left(\sigma^{2} / T\right)} \\
& \cdot \exp \left\{-\frac{\left(\overline{\mathbf{x}}_{c s}-(\alpha / T) \Lambda \mathbf{d}(\tau)\right)^{H}\left(\overline{\mathbf{x}}_{c s}-(\alpha / T) \Lambda \mathbf{d}(\tau)\right)}{\left(\sigma^{2} / T\right)}\right\}, \\
& f\left(\overline{\mathbf{x}}_{c s} \mid H_{0}\right)=\frac{1}{\pi^{M}\left(\sigma^{2} / T\right)} \exp \left\{-\frac{\overline{\mathbf{x}}_{c s}{ }^{H} \overline{\mathbf{x}}_{c s}}{\left(\sigma^{2} / T\right)}\right\} .
\end{aligned}
$$

The GLRT is then defined [26] as

$$
\xi=2 \ln \left(\frac{\max _{\alpha, \tau} f\left(\overline{\mathbf{x}}_{c s} \mid \alpha, \tau, H_{1}\right)}{f\left(\overline{\mathbf{x}}_{c s} \mid H_{0}\right)}\right) \underset{H_{0}}{\stackrel{H_{1}}{\gtrless}} \gamma .
$$

We note now that maximizing $f\left(\overline{\mathbf{x}}_{c s} \mid \alpha, \tau, H_{1}\right)$ with respect to $\alpha$ and $\tau$ is equivalent to minimizing

$$
\left(\overline{\mathbf{x}}_{c s}-\frac{\alpha}{T} \Lambda \mathbf{d}(\tau)\right)^{H}\left(\overline{\mathbf{x}}_{c s}-\frac{\alpha}{T} \Lambda \mathbf{d}(\tau)\right)
$$

which in turn is equivalent to maximizing

$$
\frac{1}{T} 2 \operatorname{Re}\left\{\alpha^{*} \mathbf{d}^{H}(\tau) \Lambda^{H} \overline{\mathbf{x}}_{c s}\right\}-\frac{1}{T^{2}}|\alpha|^{2} \mathbf{d}^{H}(\tau) \Lambda^{H} \boldsymbol{\Lambda} \mathbf{d}(\tau) .
$$

Furthermore, expression (15) is maximized for any $\tau$ by

$$
\widehat{\alpha}=\frac{T \mathbf{d}^{H}(\tau) \Lambda^{H} \overline{\mathbf{x}}_{c s}}{\mathbf{d}^{H}(\tau) \Lambda^{H} \Lambda \mathbf{d}(\tau)} .
$$

Using (11) in (13) results in the following decision rule:

$$
\xi=\max _{\tau}\left(\frac{\hat{\alpha}^{*} \mathbf{d}^{H}(\tau) \boldsymbol{\Lambda}^{H} \boldsymbol{\Lambda} \mathbf{d}(\tau) \hat{\alpha}}{\sigma^{2} T / 2}\right) \underset{H_{0}}{\stackrel{H_{1}}{\gtrless}} \gamma .
$$

\section{Compressive GLRT Detector Performance Analysis}

In this section, we first analyze the receiver operating characteristics (ROC) of compressive GLRT detector and then consider the problem of how compressive ratio affects the compressive GLRT detector.

4.1. Receiver Operating Characteristics. For compressive GLRT detector derived in Section 3, it is easy to show [26] that

$$
\begin{aligned}
& \xi_{n} \sim \begin{cases}\chi_{2}^{2} & \mathbf{H}_{0} \\
\chi_{2}^{\prime 2}(\lambda) & \mathbf{H}_{1},\end{cases} \\
& \lambda=\frac{\alpha^{H} \mathbf{d}^{H}(\tau) \Lambda^{H} \boldsymbol{\Lambda} \mathbf{d}(\tau) \alpha}{\sigma^{2} T / 2},
\end{aligned}
$$

where $\chi_{2}^{2}$ represents a chi-squared PDF with 2 degrees of freedom and $\chi_{2}^{\prime 2}(\lambda)$ represents a chi-squared PDF with 2 degrees of freedom and noncentrality parameter $\lambda$.

Let $P_{\mathrm{FA}}$ (bin) denote the false alarm rate of only one range cell we observe, and then we have

$$
P_{\mathrm{FA}}(\text { bin })=Q_{\chi_{2}^{2}}(\gamma),
$$

where $Q_{\chi_{2}^{2}}(\cdot)$ is the right-tail probability of a chi-squared random variable with 2 degrees of freedom. Since all $\xi_{n}\left(\overline{\mathbf{x}}_{c s}\right)$ is independent, therefore, the false alarm rate is

$$
\begin{aligned}
P_{\mathrm{FA}} & =\operatorname{Pr}\left\{\max _{n} \xi_{n}\left(\overline{\mathbf{x}}_{c s}\right)>\gamma ; H_{0}\right\} \\
& =1-\operatorname{Pr}\left\{\max _{n} \xi_{n}\left(\overline{\mathbf{x}}_{c s}\right)<\gamma ; H_{0}\right\} \\
& =1-\operatorname{Pr}\left\{\bigcap_{n} \xi_{n}\left(\overline{\mathbf{x}}_{c s}\right)<\gamma ; H_{0}\right\} \\
& =1-\prod_{n} \operatorname{Pr}\left\{\xi_{n}\left(\overline{\mathbf{x}}_{c s}\right)<\gamma ; H_{0}\right\},
\end{aligned}
$$

where

$$
\begin{aligned}
\operatorname{Pr}\left\{\xi_{n}\left(\overline{\mathbf{x}}_{c s}\right)<\gamma ; H_{0}\right\} & =\int_{0}^{\gamma} \frac{1}{2} \exp \left(\frac{-u}{2}\right) d u \\
& =1-\exp \left(\frac{-\gamma}{2}\right) .
\end{aligned}
$$


Hence

$$
P_{\mathrm{FA}}=1-\left(1-\exp \left(\frac{-\gamma}{2}\right)\right)^{N},
$$

where $N$ is the number of range cells we observe. For a small $P_{\mathrm{FA}}$, we have $\exp (-\gamma / 2) \ll 1$. If $x \ll 1$, then $(1-x)^{N} \approx 1-N x$, and then (23) becomes

$$
P_{\mathrm{FA}}=N \exp \left(\frac{-\gamma}{2}\right)=N P_{\mathrm{FA}}(\text { bin }),
$$

where $P_{\mathrm{FA}}$ (bin) is the false alarm rate of only one range cell we observe. Therefore, $P_{\mathrm{FA}}$ increases linearly as $N$. From (20), we can obtain

$$
\begin{aligned}
P_{\mathrm{FA}}(\text { bin }) & =Q_{\chi_{2}^{2}}(\gamma)=\exp \left(-\frac{\gamma}{2}\right) \Longrightarrow \\
\gamma & =2 \ln \frac{1}{P_{\mathrm{FA}}(\text { bin })} .
\end{aligned}
$$

Combining (24) and (25), we have the detection performance

$$
P_{D}=Q_{\chi_{2}^{\prime 2}(\lambda)}\left(2 \ln \frac{N}{P_{\mathrm{FA}}}\right),
$$

where $Q_{\chi_{2}^{\prime 2}(\lambda)}(\cdot)$ is the right-tail probability for a noncentral chi-squared random variable with 2 degrees of freedom and noncentrality parameter $\lambda$. A radar detector's performance is measured by its ability to achieve a certain probability of detection $\left(P_{D}\right)$ and probability of false alarm $\left(P_{\mathrm{FA}}\right)$ for a given SNR. Examining a radar detector's ROC curves provides insight into its performance by calculating (26).

4.2. Effect of the Compressive Ratio. In order to employ the CS theory, we now introduce a random sampling matrix $\Phi \in$ $\mathbb{R}^{M \times N}$ :

$$
\boldsymbol{\Phi}_{i j}= \begin{cases}1 & i=1,2, \ldots, M, j \in[1, N] \\ 0 & \text { others. }\end{cases}
$$

and then (7) can be rewritten as

$$
\overline{\mathbf{x}}_{c s}=\Phi\left(\alpha \Lambda_{u} \mathbf{d}_{u}(\tau)+\overline{\mathbf{w}}_{u}\right)=\Phi \overline{\mathbf{r}}
$$

where

$$
\begin{aligned}
\overline{\mathbf{r}} & =\alpha \boldsymbol{\Lambda}_{u} \mathbf{d}_{u}(\tau)+\overline{\mathbf{w}}_{u} \\
\mathbf{d}_{u}(\tau) & =\left[1, e^{-j 2 \pi \tau / T}, \ldots, e^{-j 2 \pi(N-1) \tau / T}\right]^{T}, \\
\overline{\mathbf{w}}_{u} & =\left[\bar{w}_{c s}[0], \bar{w}_{c s}[1], \ldots, \bar{w}_{c s}[N-1]\right]^{T},
\end{aligned}
$$

and $\boldsymbol{\Lambda}_{u}=\operatorname{diag}\left\{\overline{\mathbf{s}}_{u}\right\}$, where

$$
\overline{\mathbf{s}}_{u}=[\bar{s}[0], \bar{s}[1], \ldots, \bar{s}[N-1]]^{T} .
$$

Following the same derivation as in Section 4.1, (19) can be rewritten as

$$
\lambda=\frac{\alpha^{H} \mathbf{d}_{u}^{H}(\tau) \Lambda_{u}^{H} \boldsymbol{\Phi}^{H}\left(\boldsymbol{\Phi} \boldsymbol{\Phi}^{H}\right)^{-1} \boldsymbol{\Phi}_{u} \mathbf{d}_{u}(\tau) \alpha}{\sigma^{2} T / 2} .
$$

We define

$$
\mathbf{P}_{\boldsymbol{\Phi}}=\boldsymbol{\Phi}^{H}\left(\boldsymbol{\Phi} \boldsymbol{\Phi}^{H}\right)^{-1} \boldsymbol{\Phi}
$$

as the orthogonal projection operator onto $R(\Phi)$, that is, the row space of $\boldsymbol{\Phi}$. Therefore, (31) becomes

$$
\lambda=2 \cdot \frac{(1 / T)\left\|\mathbf{P}_{\Phi} \Lambda_{u} \mathbf{d}_{u}(\tau) \alpha\right\|_{2}}{\sigma^{2}} .
$$

Let us now define

$$
\mathrm{SNR}=\frac{(1 / T)\left\|\boldsymbol{\Lambda}_{u} \mathbf{d}_{u}(\tau) \alpha\right\|_{2}^{2}}{\sigma^{2}}
$$

as signal-to-noise ratio (SNR). Let $\delta \in(0,1)$; we can bound the performance of the compressive GLRT detector as follows.

Theorem 1. Suppose that $\sqrt{N / M} \mathbf{P}_{\Phi}$ provides a $\delta$-stable embedding of $(S,\{0\})$. Then for any $s \in S$, one can detect $s$ with error rate

$$
\begin{aligned}
& P_{D} \leq Q_{\chi_{2}^{\prime 2}(2 \cdot(1+\delta)(M / N) \mathrm{SNR})}\left(2 \ln \frac{N}{P_{\mathrm{FA}}}\right), \\
& P_{D} \geq Q_{\chi_{2}^{\prime 2}(2 \cdot(1-\delta)(M / N) \mathrm{SNR})}\left(2 \ln \frac{N}{P_{\mathrm{FA}}}\right) .
\end{aligned}
$$

Proof. By our assumption that $\sqrt{N / M} \mathbf{P}_{\boldsymbol{\Phi}}$ provides a $\delta$-stable embedding of $(S,\{0\})$, we know from Equation (5) of [17] that

$$
\begin{aligned}
\sqrt{1-\delta}\left\|\boldsymbol{\Lambda}_{u} \mathbf{d}_{u}(\tau) \alpha\right\|_{2} & \leq \sqrt{\frac{N}{M}}\left\|\mathbf{P}_{\Phi} \boldsymbol{\Lambda}_{u} \mathbf{d}_{u}(\tau) \alpha\right\|_{2} \\
& \leq \sqrt{1+\delta}\left\|\boldsymbol{\Lambda}_{u} \mathbf{d}_{u}(\tau) \alpha\right\|_{2} .
\end{aligned}
$$

Furthermore, we have

$$
\begin{aligned}
& (1-\delta) \frac{M}{N}\left\|\boldsymbol{\Lambda}_{u} \mathbf{d}_{u}(\tau) \alpha\right\|_{2}^{2} \leq\left\|\mathbf{P}_{\Phi} \boldsymbol{\Lambda}_{u} \mathbf{d}_{u}(\tau) \alpha\right\|_{2}^{2} \\
& \leq(1+\delta) \frac{M}{N}\left\|\boldsymbol{\Lambda}_{u} \mathbf{d}_{u}(\tau) \alpha\right\|_{2}^{2} .
\end{aligned}
$$

Specifically, for typical values of $\delta$, we have

$$
\left\|\mathbf{P}_{\Phi} \boldsymbol{\Lambda}_{u} \mathbf{d}_{u}(\tau) \alpha\right\|_{2}^{2} \approx \frac{M}{N}\left\|\boldsymbol{\Lambda}_{u} \mathbf{d}_{u}(\tau) \alpha\right\|_{2}^{2} .
$$

Combining (39) and (34), (33) reduces to

$$
\begin{aligned}
\lambda & =2 \cdot \frac{(1 / T)\left\|\mathbf{P}_{\Phi} \boldsymbol{\Lambda}_{u} \mathbf{d}_{u}(\tau) \alpha\right\|_{2}}{\sigma^{2}} \\
& \approx 2 \cdot \frac{M}{N} \frac{(1 / T)\left\|\boldsymbol{\Lambda}_{u} \mathbf{d}_{u}(\tau) \alpha\right\|_{2}}{\sigma^{2}}=2 \cdot \frac{M}{N} \text { SNR. }
\end{aligned}
$$

Finally, (26) becomes

$$
P_{D} \approx Q_{\chi_{2}^{\prime 2}(2 \cdot(M / N) \text { SNR })}\left(2 \ln \frac{N}{P_{\mathrm{FA}}}\right) .
$$

From (41), one can find that the probability of detection $\left(P_{D}\right)$ increases as the compressive ratio (i.e., $M / N$ ) increases for a given probability of false alarm $\left(P_{\mathrm{FA}}\right)$. Furthermore, $(41)$ can be used to quantify the effect of sub-Nyquist sampling on the detection performance. 


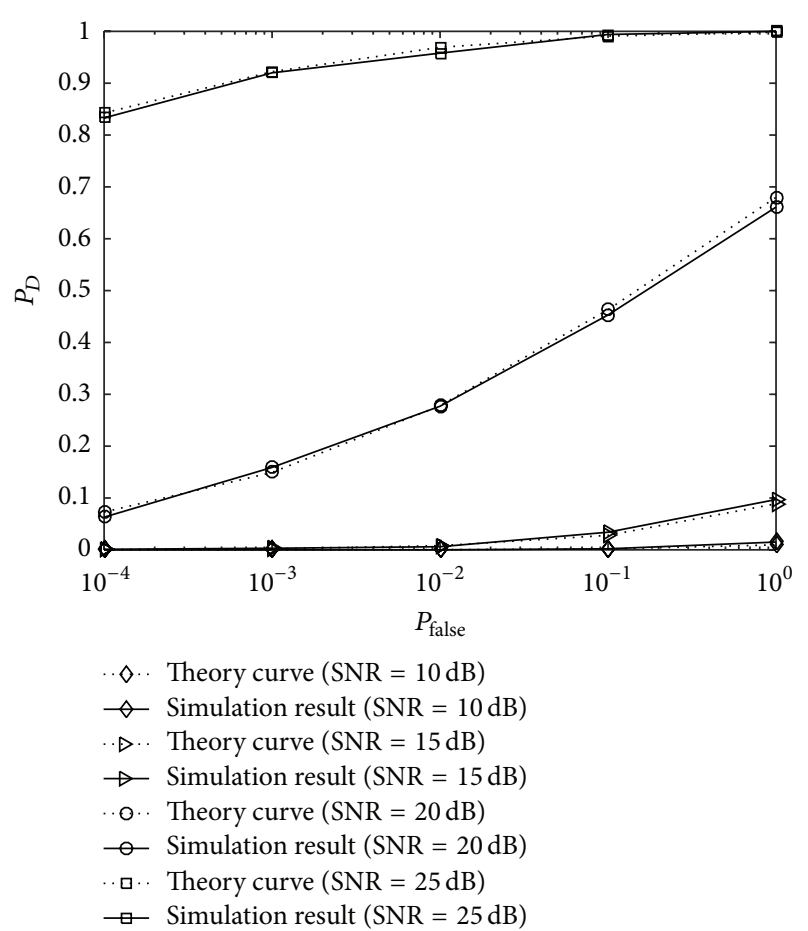

FIGURE 2: ROC curves near the theory curve of ROC for different SNR.

\section{Simulation Results}

In this section, we discuss simulation results and compare the performance of the different systems and setups. In order to first demonstrate the effectiveness of predicted detection performance we resort to Monte Carlo simulations, where we consider a target with $\alpha$ constant in (1). Further assume the round trip delay of the target is $1.1 \mu \mathrm{s}$. The other parameters used are $T=10 \mu \mathrm{s}, B=100 \mathrm{MHz}$.

5.1. Receiver Operating Characteristics. The set of simulation parameters is compressive ratio $M / N=0.2$ over 10000 Monte Carlo simulations. Figure 2 shows the ROC of the subNyquist radar systems for different SNR, which are also contrasted with that theory curve predicted by (26). It is observed that the ROC curves obtained by Monte Carlo simulation are tightly concentrated around the expected performance curve described in (26) with high probability in all these cases.

In order to demonstrate the predicted theory bounds of detection performance in Section 4, we repeat the experiment in Figure 2 for different SNR. Over 10000 Monte Carlo simulations, the ROC curves are illustrated in Figure 3 and are contrasted to the upper bound and lower bound of detection performance given by (35) and (36), respectively, where the $\delta$ parameter is set equal to an empirical value 0.05 . It is observed that the ROC curves obtained by Monte Carlo simulation are tightly concentrated between the expected upper bound and lower bound in all these cases.

5.2. Compressive Ratio. We next investigate the effect of the compressive ratio $M / N$ on the performance of the

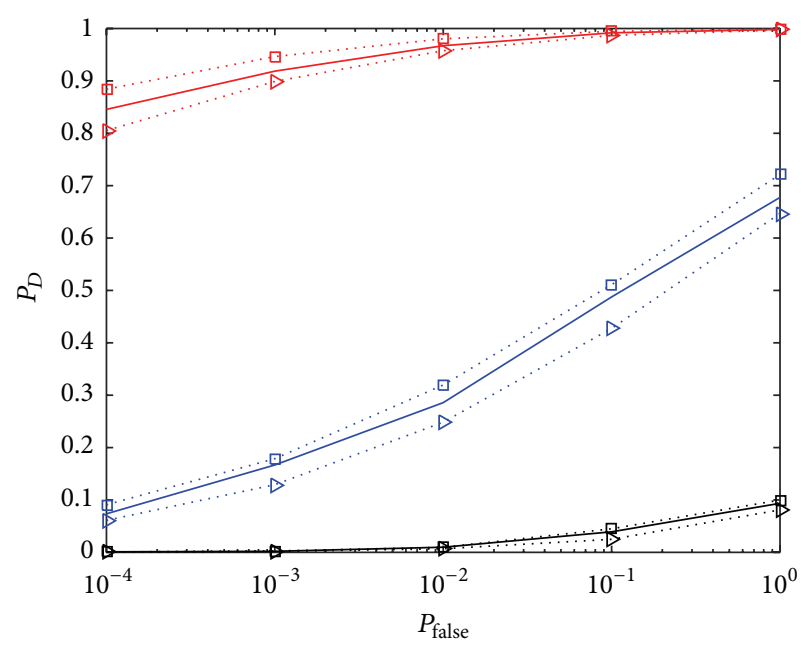

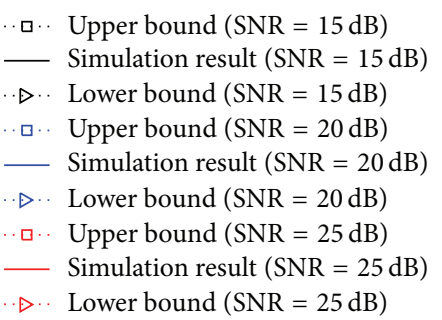

FIgURE 3: Concentration of ROC curve around the expected performance curve.

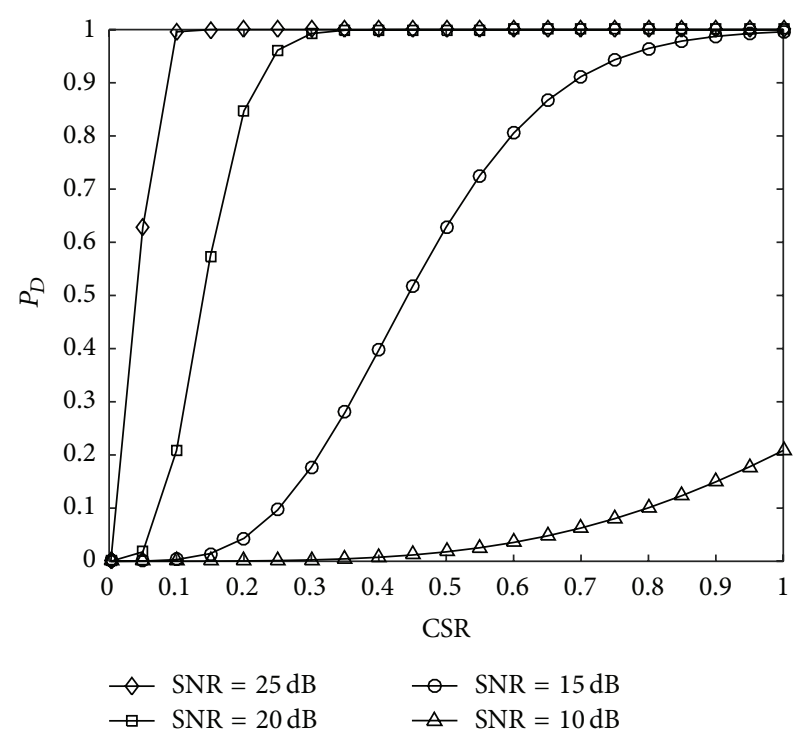

FIGURE 4: Effect of compressive ratio on detection performance of compressive GLRT detector.

compressive detector. Figure 4 illustrated the exponential increase in the detection probability as more measurements are taken, which plots the performance obtained over 10000 Monte Carlo simulation experiments for a range of SNRs with $P_{\text {false }}=10^{-4}$. From Figure 4 , we can see that the detection probability $P_{D}$ should approach 1 exponentially fast as compressive ratio is increased. 


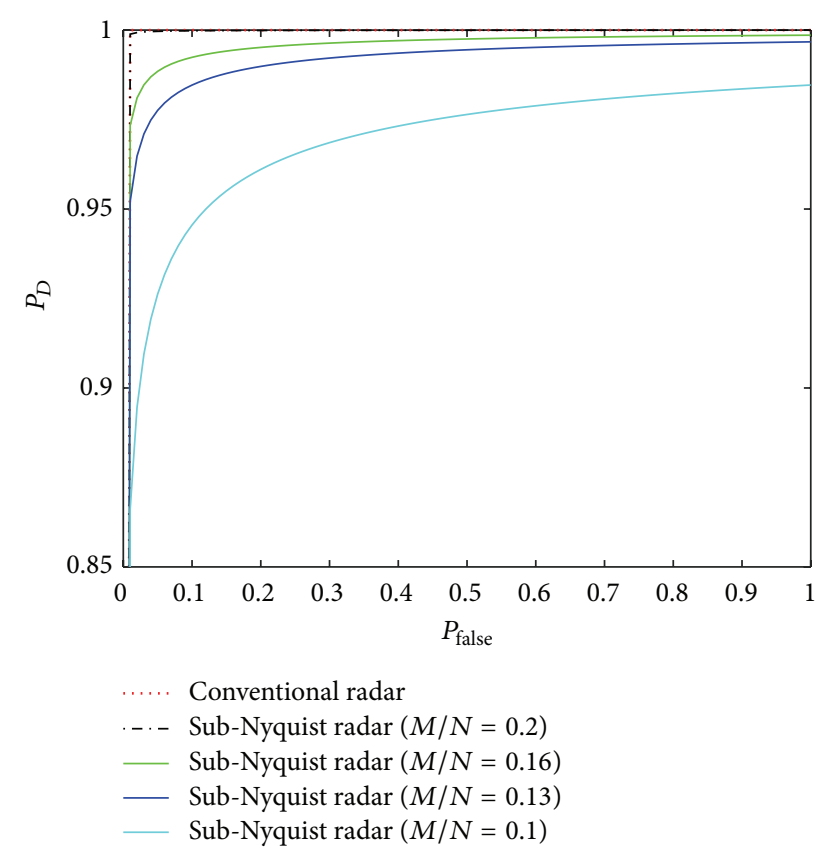

FIgURE 5: Comparison of detection performance between subNyquist radar and conventional radar.

5.3. Comparing Sub-Nyquist and Conventional Radars. In this subsection, we give more insight into the sub-Nyquist and conventional radar detectors by comparing the ROC curves. As illustrated in Figure 5, in certain cases (relatively high SNR; $22 \mathrm{~dB}$ in this simulation experiment) the compressive GLRT detector can perform almost as well as the traditional GLRT detector with a very small fraction of the number of measurements required by traditional detection. It is observed that the ROC curve approaches the upper-left corner as the compressive ratio increases, which means that we can achieve very high detection probability while simultaneously keeping the false alarm rate very low.

Here, we compare the different radar systems according to their computation time. Assume the SNR is $12 \mathrm{~dB}$, and the false alarm probability is $10^{-4}$. Figure 6 shows the averaging computation time curves for sub-Nyquist radar system and conventional radar system as compressive ratio increasing over 100 independent simulation experiments. It is observed that the computation time curve for sub-Nyquist radar is on the bottom of Figure 6, and the computation time of sub-Nyquist radar linearly increases with the compressive ratio, which shows that the sub-Nyquist radar can provide for saving computation time over conventional radar.

From Figures 5 and 6, we can see that a good detection performance can be obtained only with fewer measurements. Simulation results imply that it is possible to achieve comparable performance with less computational load and less computation time for sub-Nyquist radar.

\section{Conclusion}

The growing demands for target distinction capability and spatial resolution imply significant growth in the bandwidth

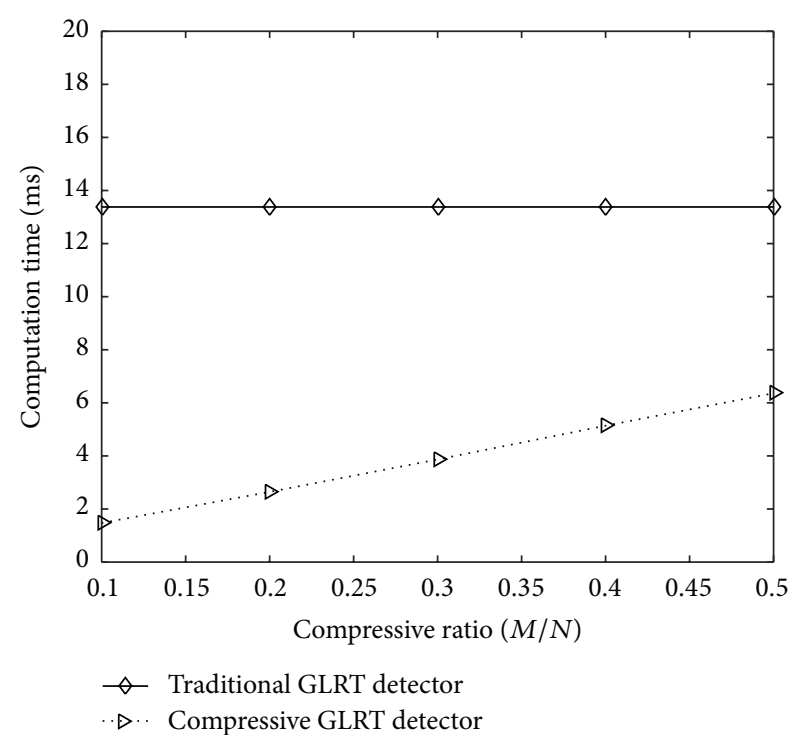

Figure 6: Comparison of computation time between sub-Nyquist radar and conventional radar.

of the transmitted pulse in modern radar systems. Under the confinement of classic Shannon-Nyquist sampling theorem, it requires that the received signals must first be sampled at twice the baseband bandwidth in order to keep the signal not aliasing, which caused huge computation burden. In addition, real-time processing of data typically results in high power consumption. As new approaches for radar sensing based on the finite rate of innovation (FRI) and Xampling frameworks, sub-Nyquist radar system allows sampling of radar signals at rates much lower than Nyquist, which has been demonstrated by real-time analog experiments in hardware. These techniques allow significant reduction in sampling rate, implying potential power saving, and hence gaining substantial storage capacity reduction for subsequent processing.

This paper investigates a sub-Nyquist radar approach employing multichannel direct sampling of the Fourier series coefficients for the detection of a single target. In this paper, a compressive GLRT detector is presented for sub-Nyquist radar directly based on the compressive measurements and without ever reconstructing the signal involved. We analyze the compressive GLRT detection performance and provide its theoretical bounds. The compressive GLRT detection performance is also contrasted with that of a conventional radar approach, which employs traditional ADC at Nyquist sampling rates. Simulation results show that the compressive GLRT detector can perform almost as well as the traditional GLRT detector with a very small fraction of the number of measurements required by traditional detection in relatively high SNR cases. Both the theoretical conclusion and simulation results demonstrate that the compressive detection approach for sub-Nyquist radar is very suitable especially for large bandwidth radar system in real-time processing. 


\section{Competing Interests}

The authors declare that there are no competing interests regarding the publication of this paper.

\section{Acknowledgments}

This work is supported by the Science and Technical Achievements Resulted from Cooperation of Industry, Education and Academy Project of Guangdong Province (no. 2016B090918084), the Pre-Research Fund of General Armament Department (no. 51326020602), the Science \& Technology Innovation Project of Shenzhen (nos. JCYJ20150324141711674, JCYJ20160307112710376), National Natural Science Foundation of China (no. 61401478), and the Natural Science Foundation of SZU (no. 2016056).

\section{References}

[1] D. L. Donoho, "Compressed sensing," IEEE Transactions on Information Theory, vol. 52, no. 4, pp. 1289-1306, 2006.

[2] E. J. Candés, J. Romberg, and T. Tao, "Robust uncertainty principles: exact signal reconstruction from highly incomplete frequency information," IEEE Transactions on Information Theory, vol. 52, no. 2, pp. 489-509, 2006.

[3] M. F. Duarte, M. A. Davenport, M. B. Wakin, and R. G. Baraniuk, "Sparse signal detection from incoherent projections," in Proceedings of the IEEE International Conference on Acoustics, Speech and Signal Processing (ICASSP '06), vol. 3, pp. 305-308, Toulouse, France, May 2006.

[4] E. J. Candes and M. B. Wakin, "An introduction to compressive sampling," IEEE Signal Processing Magazine, vol. 25, no. 2, pp. 21-30, 2008.

[5] R. G. Vaughan, N. L. Scott, and D. R. White, "The theory of bandpass sampling," IEEE Transactions on Signal Processing, vol. 39, no. 9, pp. 1973-1984, 1991.

[6] R. Baraniuk and P. Steeghs, "Compressive radar imaging," in Proceedings of the IEEE Radar Conference, pp. 128-133, IEEE, Boston, Mass, USA, April 2007.

[7] M. Herman and T. Strohmer, "Compressed sensing radar," in Proceedings of the IEEE Radar Conference (RADAR '08), pp. 16, Rome, Italy, May 2008.

[8] M. A. Herman and T. Strohmer, "High-resolution radar via compressed sensing," IEEE Transactions on Signal Processing, vol. 57, no. 6, pp. 2275-2284, 2009.

[9] J. H. G. Ender, “On compressive sensing applied to radar," Signal Processing, vol. 90, no. 5, pp. 1402-1414, 2010.

[10] F. Xi, S. Chen, and Z. Liu, "Quadrature compressive sampling for radar signals," IEEE Transactions on Signal Processing, vol. 62, no. 11, pp. 2787-2802, 2014.

[11] N. S. Subotic, B. Thelen, K. Cooper et al., "Distributed RADAR waveform design based on compressive sensing considerations," in Proceedings of the IEEE Radar Conference (RADAR '08), pp. 1-6, IEEE, Rome, Italy, May 2008.

[12] C. R. Berger, S. Zhou, and P. Willett, "Signal extraction using compressed sensing for passive radar with OFDM signals," in Proceedings of the 11th International Conference on Information Fusion (FUSION '08), pp. 1-6, IEEE, Cologne, Germany, July 2008.

[13] J. D. Zhang, D. Zhu, and G. Zhang, "Adaptive compressed sensing radar oriented toward cognitive detection in dynamic sparse target scene," IEEE Transactions on Signal Processing, vol. 60, no. 4, pp. 1718-1729, 2012.

[14] Y. Yu, A. P. Petropulu, and H. V. Poor, "MIMO radar using compressive sampling," IEEE Journal on Selected Topics in Signal Processing, vol. 4, no. 1, pp. 146-163, 2010.

[15] C.-Y. Chen and P. P. Vaidyanathan, "Compressed sensing in MIMO radar," in Proceedings of the 42nd Asilomar Conference on Signals, Systems and Computers (ASILOMAR '08), pp. 41-44, Pacific Grove, Calif, USA, October 2008.

[16] J. Tropp and A. C. Gilbert, "Signal recovery from partial information via orthogonal matching pursuit," IEEE Transactions on Information Theory, vol. 53, no. 12, pp. 4655-4666, 2006.

[17] M. A. Davenport, P. T. Boufounos, M. B. Wakin, and R. G. Baraniuk, "Signal processing with compressive measurements," IEEE Journal on Selected Topics in Signal Processing, vol. 4, no. 2, pp. 445-460, 2010.

[18] A. Hariri and M. Babaie-Zadeh, "Joint compressive single target detection and parameter estimation in radar without signal reconstruction," IET Radar, Sonar \& Navigation, vol. 9, no. 8, pp. 948-955, 2015.

[19] Z. Wang, G. R. Arce, and B. M. Sadler, "Subspace compressive detection for sparse signals," in Proceedings of the IEEE International Conference on Acoustics, Speech and Signal Processing (ICASSP '08), pp. 3873-3876, Las Vegas, Nev, USA, April 2008.

[20] K. Ahmed, S. Kothuri, M. Patwary, and M. Abdel-Maguid, "Subspace compressive GLRT detector for airborne MIMO radar," in Proceedings of the 16th Asia-Pacific Conference on Communications (APCC '10), pp. 302-306, IEEE, Auckland, New Zealand, November 2010.

[21] Z. Yang, X. Li, H. Wang, and R. Fa, "Knowledge-aided STAP with sparse-recovery by exploiting spatio-temporal sparsity," IET Signal Processing, vol. 10, no. 2, pp. 150-161, 2016.

[22] Z. Yang, Y. Qin, R. C. de Lamare, H. Wang, and X. Li, "Sparsitybased direct data domain space-time adaptive processing with intrinsic clutter motion," Circuits, Systems, and Signal Processing, 2016.

[23] E. Baransky, G. Itzhak, N. Wagner, I. Shmuel, E. Shoshan, and Y. Eldar, "Sub-Nyquist radar prototype: hardware and algorithm," IEEE Transactions on Aerospace and Electronic Systems, vol. 50, no. 2, pp. 809-822, 2014.

[24] K. Gedalyahu, R. Tur, and Y. C. Eldar, "Multichannel sampling of pulse streams at the rate of innovation," IEEE Transactions on Signal Processing, vol. 59, no. 4, pp. 1491-1504, 2011.

[25] O. Bar-Ilan and Y. C. Eldar, "Sub-Nyquist radar," in Proceedings of the 9th International ITG Conference on Systems, Communication and Coding (SCC '13), January 2013.

[26] S. M. Kay, Fundamentals of Statistical Signal Processing Detection Theory, vol. 2, Prentice Hall PTR, Upper Saddle River, NJ, USA, 1998. 


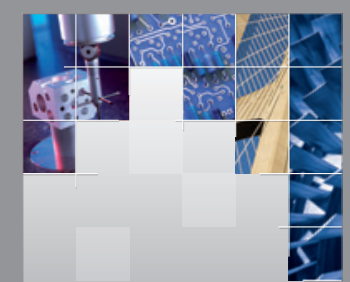

\section{Enfincering}
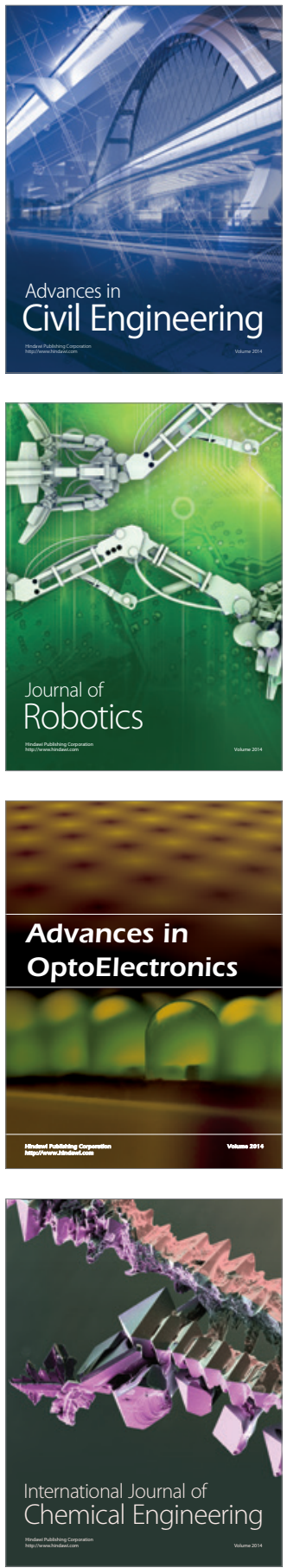

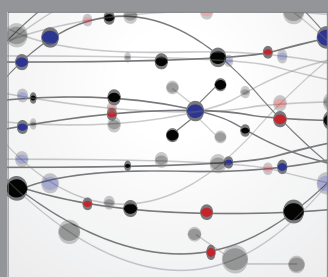

The Scientific World Journal

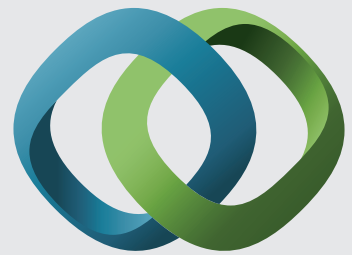

\section{Hindawi}

Submit your manuscripts at

http://www.hindawi.com
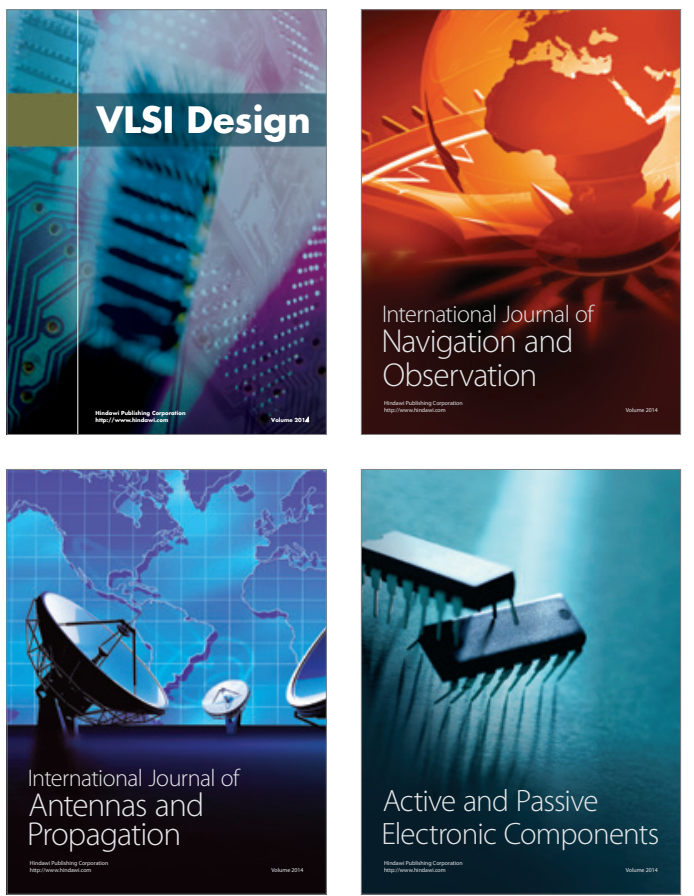
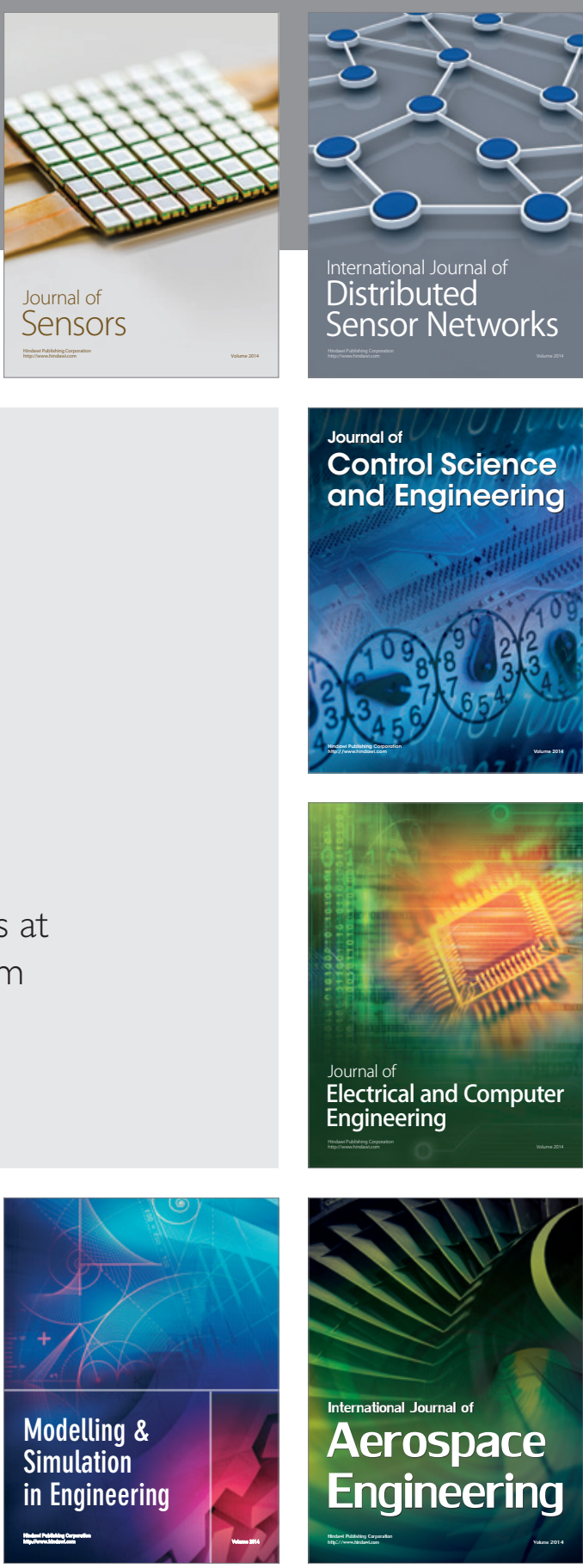

International Journal of

Distributed

Sensor Networks

Journal of

Control Science

and Engineering
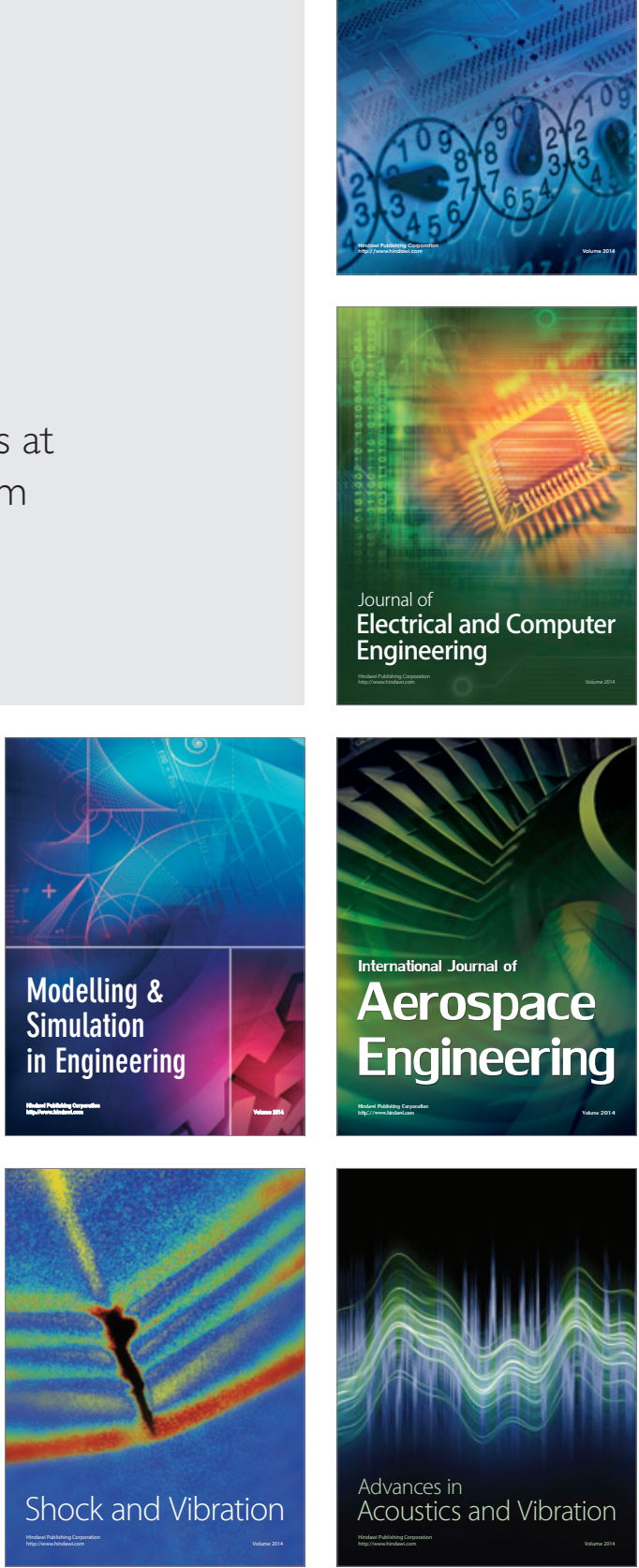